\title{
Structural Insights into Endobiotic Reactivation by Human Gut Microbiome-Encoded Sulfatases
}

Samantha M. Ervin ${ }^{1}$, Joshua B. Simpson ${ }^{1}$, Morgan E. Gibbs ${ }^{1}$, Benjamin C. Creekmore ${ }^{1}$, Lauren Lim $^{1}$, William G. Walton ${ }^{1}$, Raad Z. Gharaibeh ${ }^{2}$, and Matthew R. Redinbo ${ }^{1,3 *}$

${ }^{1}$ Department of Chemistry, University of North Carolina at Chapel Hill, Chapel Hill, NC 27599, USA

${ }^{2}$ Department of Medicine, University of Florida, Gainesville, FL 32603, USA

${ }^{3}$ Integrated Program for Biological and Genome Sciences, and Departments of Biochemistry and Microbiology, University of North Carolina at Chapel Hill, Chapel Hill, North Carolina 27599, USA

*Corresponding Author: redinbo@unc.edu 


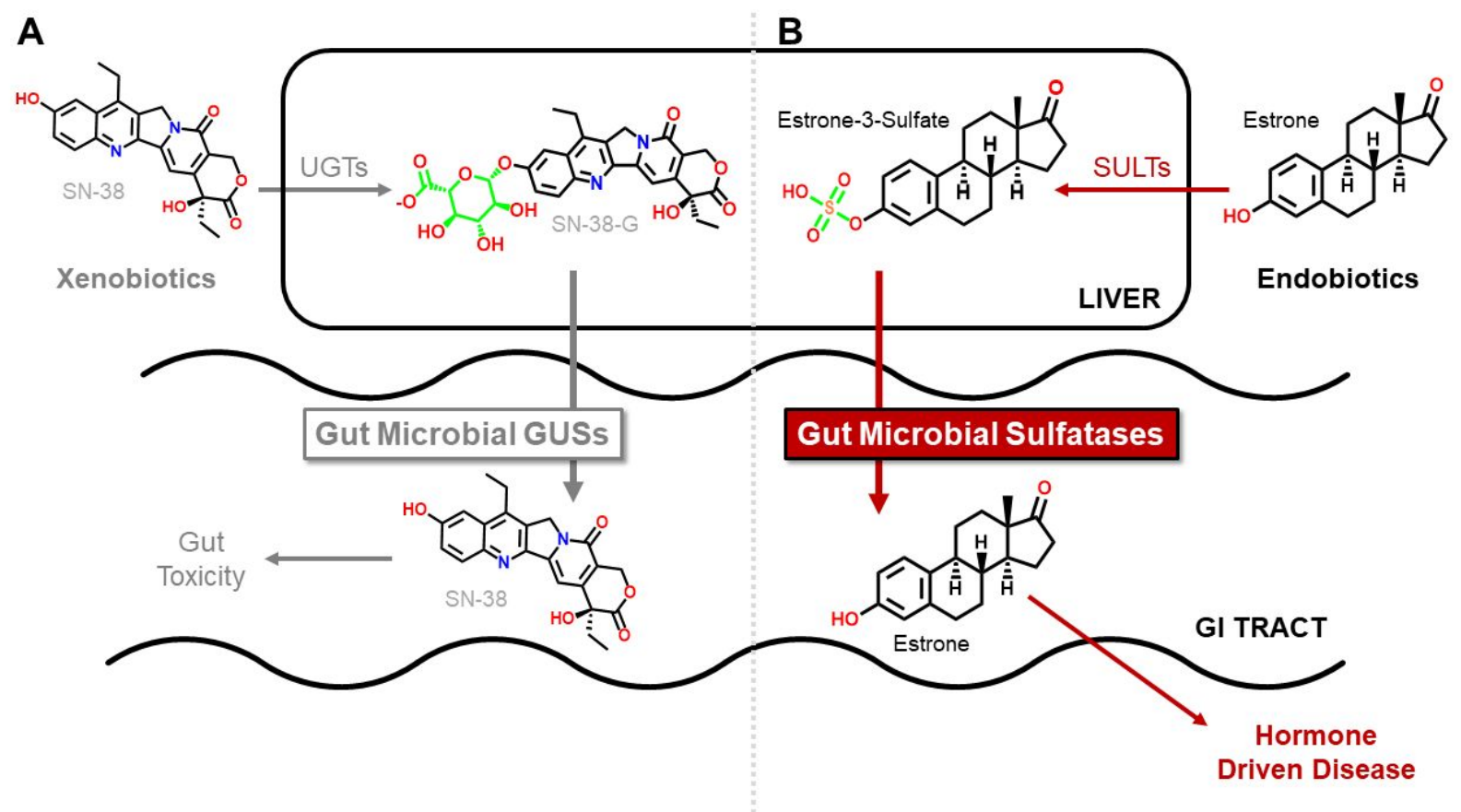

Figure S1. Reactivation of Host Phase II Metabolites by Gut Microbial Enzymes. A. Xenobiotics more commonly undergo Phase II drug metabolism mediated by UDPglucuronosyltransferases (UGTs) that append a glucuronic acid sugar (green) to compounds marked for excretion to the intestines, as shown here for the active metabolite SN-38 of the anticancer drug irinotecan. Gut microbial beta-glucuronidase enzymes (GUSs) remove the sugar as a carbon source and reactivate the drug in the gut lumen, leading to intestinal toxicity. B. Endobiotic compounds are more typically processed by the other primary form of Phase II drug metabolism - sulfotransferase enzymes (SULTs) that append a sulfate moiety to compounds prior to excretion to the intestines, as shown here for estrone. Gut microbial sulfatases remove the sulfate and reactivate in the gut the original endobiotic, which can then undergo enterohepatic recirculation to potentially drive states of disease such as hormone-positive malignancies. 
A
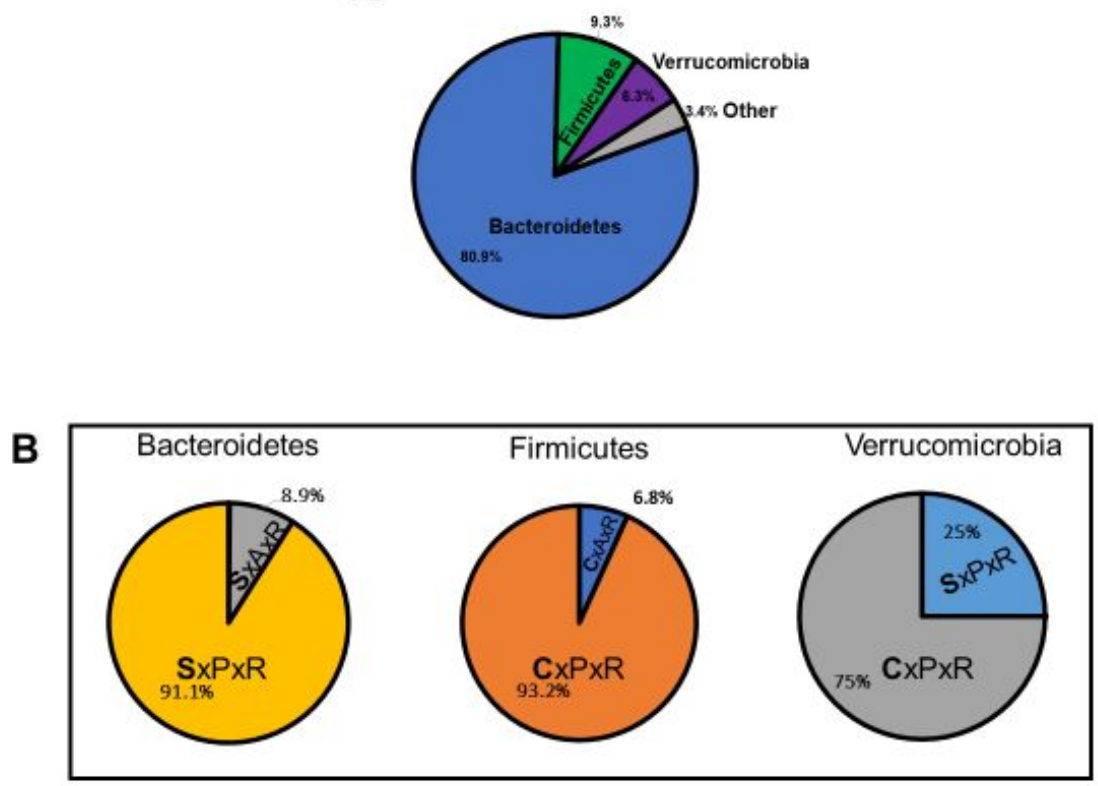

Figure S2. Sulfatase Taxonomy in HMP Stool Sample Catalogue. A. Microbial phyla. B. Catalytic motifs in each phylum with catalytic residue converted to fGly in bold. 

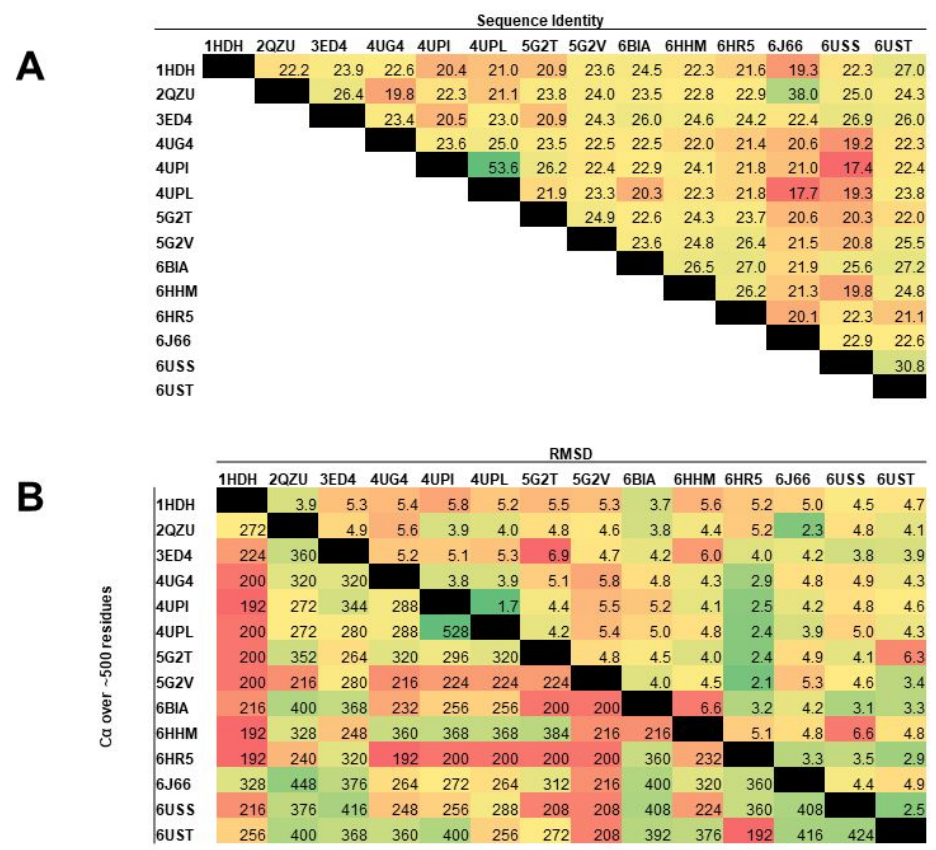

Figure S3. A. Comparison of Microbial Sulfatases in the PDB. A. Sequence identities (\%) between the 14 microbial sulfatases in the PDB. B. RMSD values over indicated number of $\mathrm{C} \alpha$ positions. RMSDs range between 2.3 and 6.9 $\dot{\mathrm{A}}$. Number of $\mathrm{C} \alpha$ positions range between 192 and 448 ; the average number of $\mathrm{C} \alpha$ positions in microbial sulfatases is $\sim 500$. 


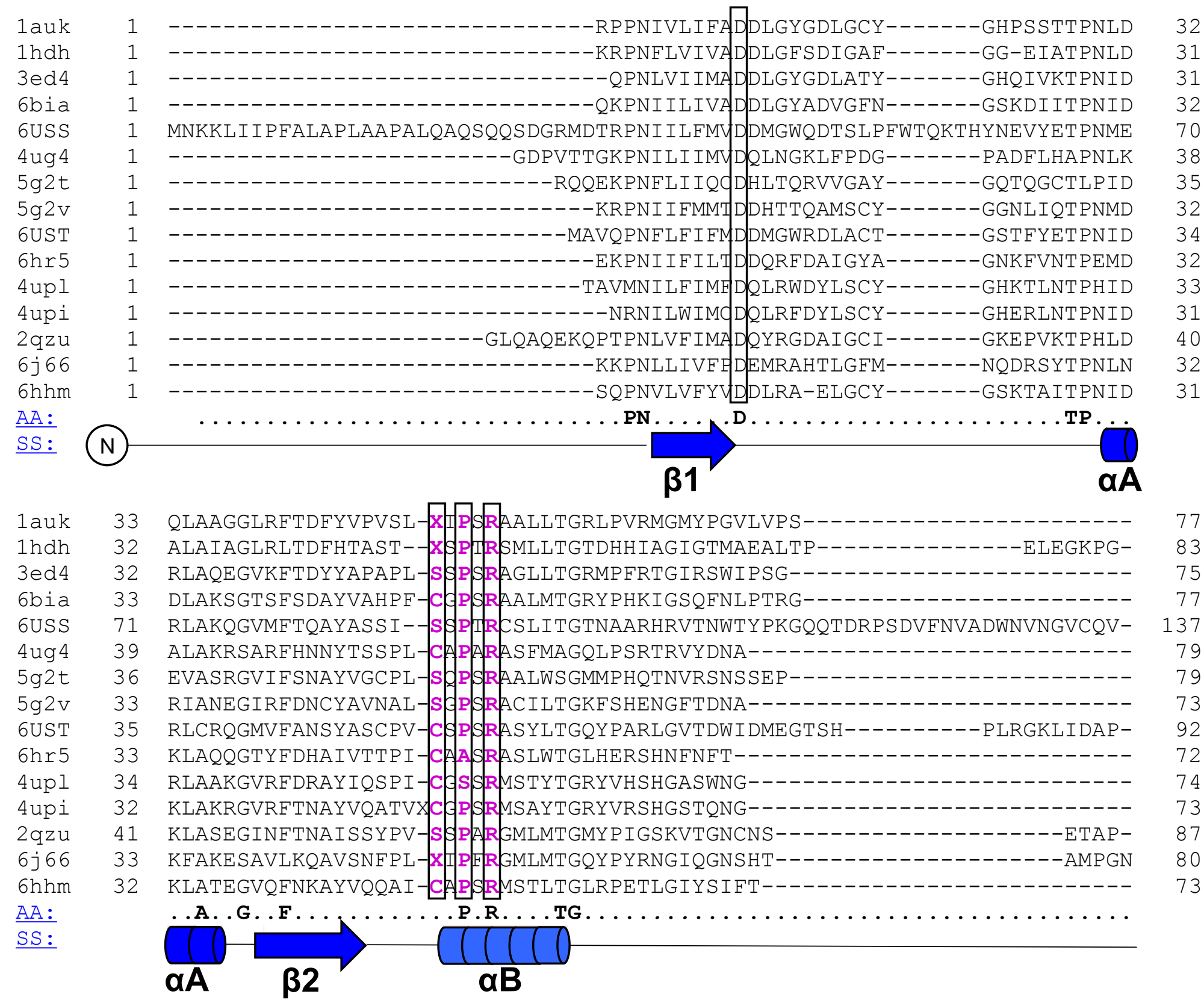




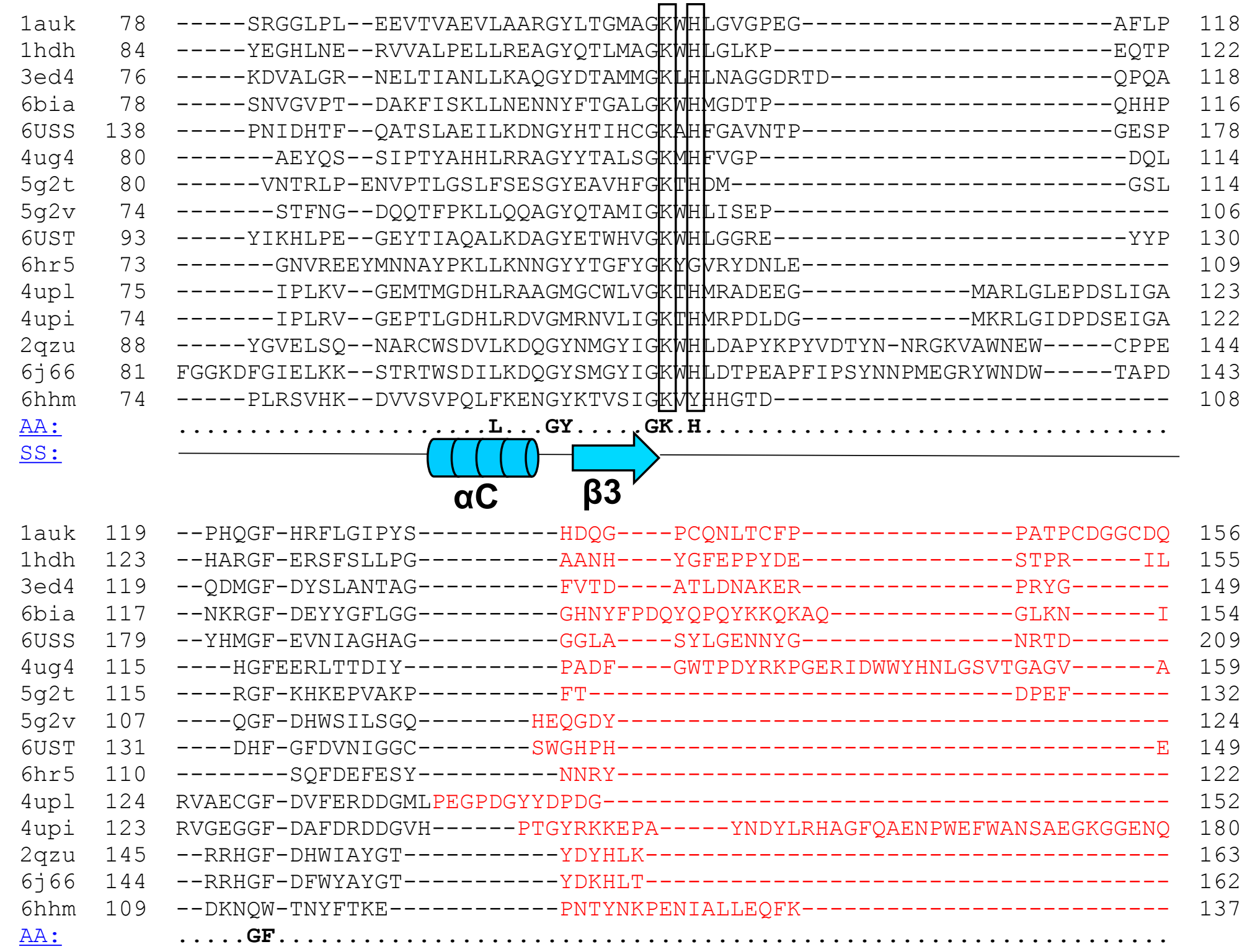

Loop 2 


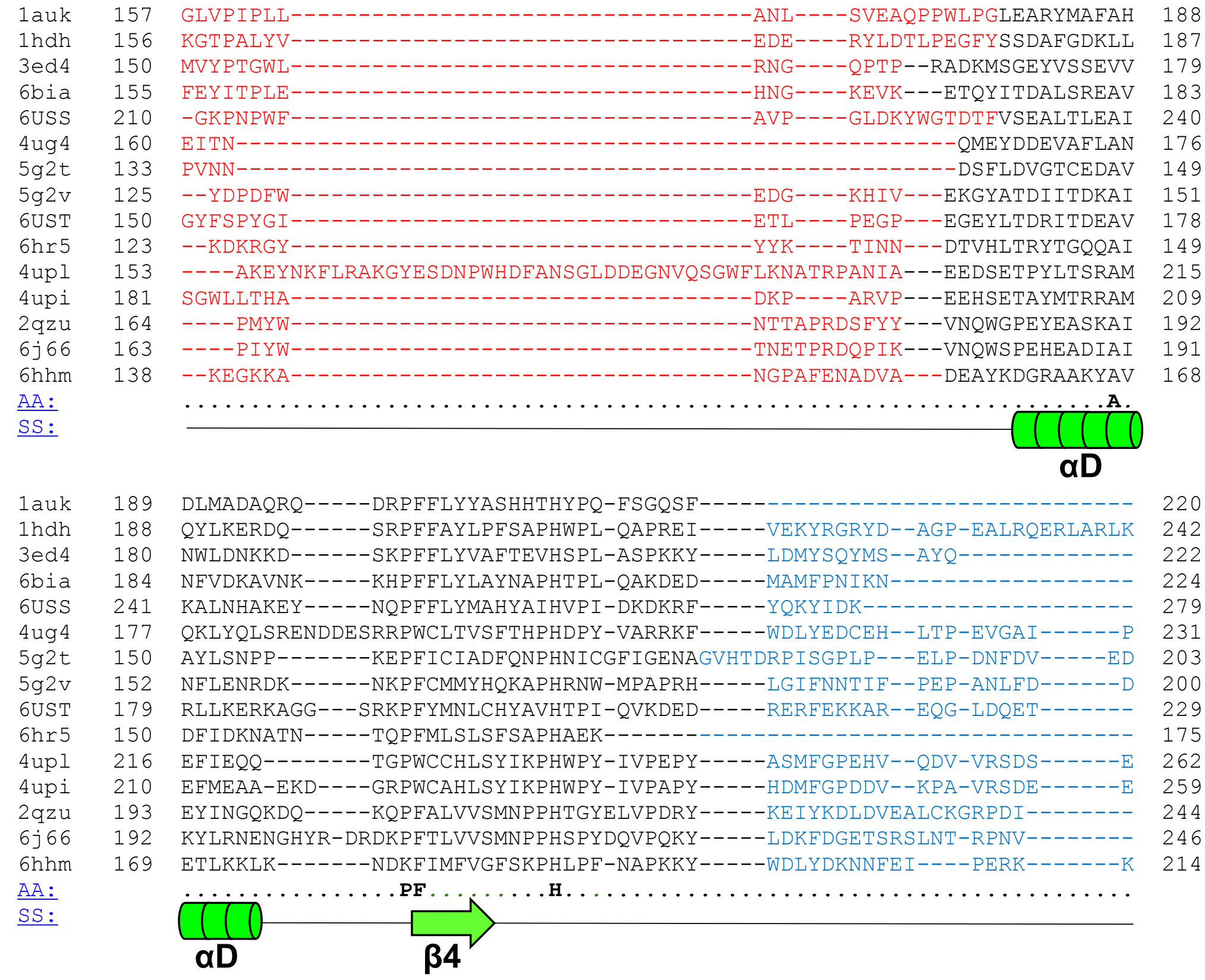


1 auk

1 hdh

3ed

$6 \mathrm{bia}$

6USS

4 ug 4

$5 g 2 t$

$5 \mathrm{~g} 2 \mathrm{v}$

$6 \mathrm{UST}$

$6 h r 5$

4 upl

4 upi

2qzu

$6 j 66$

$6 \mathrm{hhm}$

AA:

SS:

lauk

1 hdh

3ed 4

6bia

6USS

4 ug 4

$5 g 2 t$

$5 \mathrm{~g} 2 \mathrm{~V}$

$6 \mathrm{UST}$

6 hr 5

4 upl

4 upi

2qzu

$6 j 66$

$6 \mathrm{hhm}$

AA:

SS:

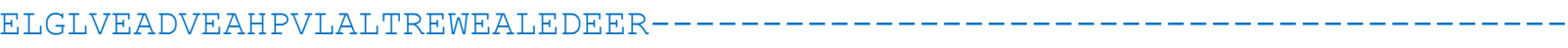
----------------- KQHPLLFYGDWA---------------------------------------

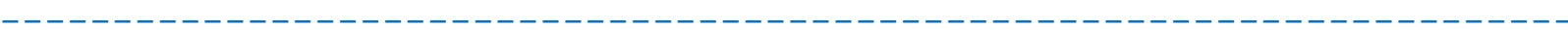

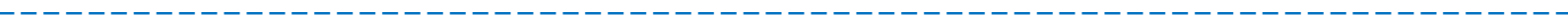
LDEQDP-----HSQRIMLSCDY----- $\mathrm{QNF}----------------------------------------$ WSNIPT-----PVQYICCSHR---RMTQAA---------------------------------------YEGRGK-----AAREQDMS IE-----HTLTNDWDLKLLTREEMLKDTTNRLYSVYKRMP SEVQDKWDSAY ----------------ALVEGEFHHTEDKK------------------------------------

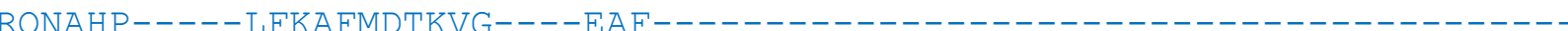

LKAAHP-----LFKAMTEEVYS----RNE--------------------------------------

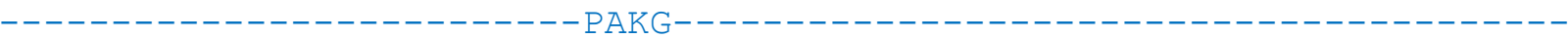
$--------------------------\mathrm{Q} W \mathrm{~W} \mathrm{-}----------------------------------------$ PENMYR-----LAL-TNW-GELKGYHGIPND----------------------------- - - - - - - -

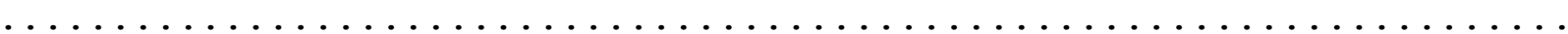

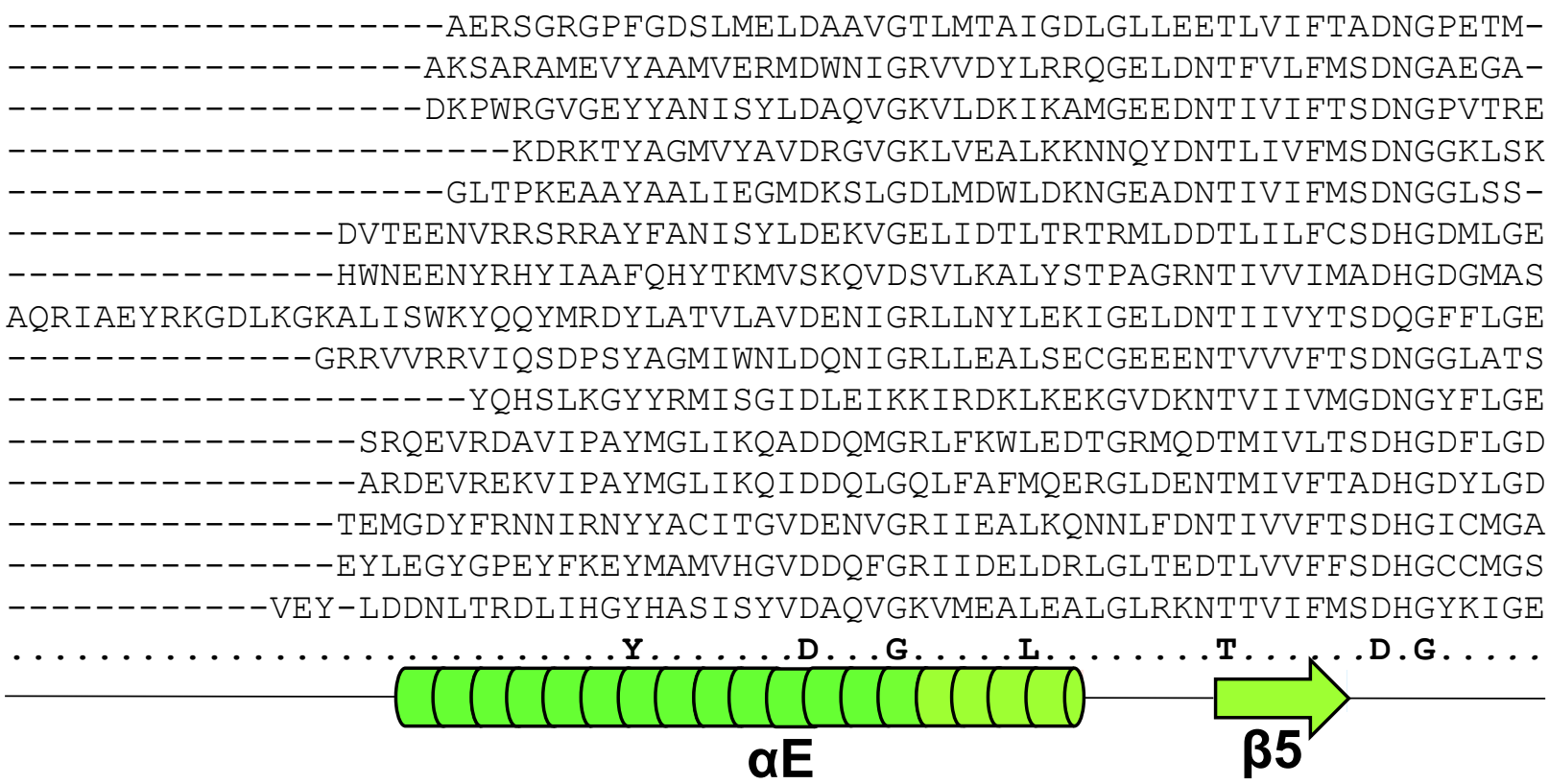




$\begin{array}{ll}\text { lauk } & 270 \\ \text { lhdh } & 322 \\ \text { 3ed4 } & 286 \\ \text { 6bia } & 272 \\ \text { 6usS } & 329 \\ \text { 4ug4 } & 307 \\ \text { 5g2t } & 281 \\ \text { 5g2v } & 331 \\ \text { 6UST } & 300 \\ \text { 6hr5 } & 225 \\ \text { 4upl } & 337 \\ \text { 4upi } & 334 \\ \text { 2qzu } & 304 \\ \text { 6j66 } & 306 \\ \text { 6hhm } & 296 \\ \text { AA: } & \end{array}$

SS:

lauk

Ihdh

3ed 4

$6 \mathrm{bia}$

6USS

4ug 4

$5 \mathrm{~g} 2 \mathrm{t}$

$5 \mathrm{~g} 2 \mathrm{~V}$

$6 \mathrm{UST}$

6 hr 5

4 upl

4 upi

2qzu

$6 j 66$

$6 \mathrm{hhm}$

$\underline{\mathrm{AA}:}$

SS:
---- RMSRG-----------------------------GCSGLLRCGKG-TTYEGGVREPA -----LLEAFPKFGPDLLGFLDRHYDNS LENI GRANSYVWYGPRWAQAATAPSRLYKA-FTTQGGIRVPA

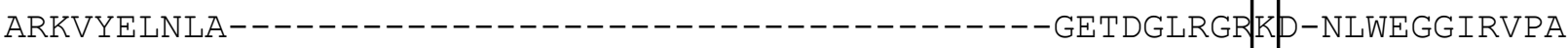

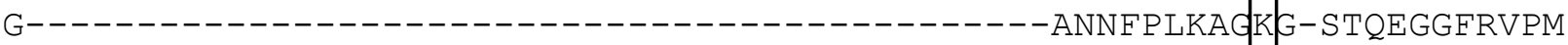
-----EPGWRD-------------------------GKLHTQNSPLNSGKG-SAYEGGVREPM

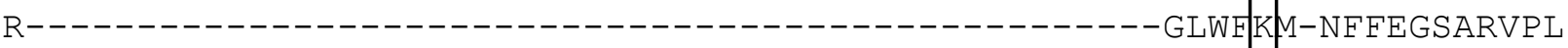
H-- --- - - - - - - H-- - - - - - - - - - - -

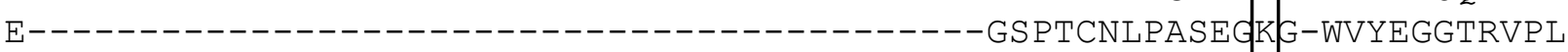

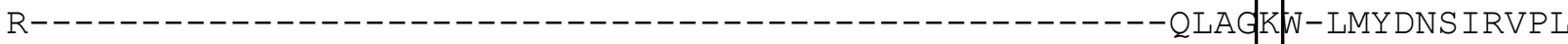
H-- - - - - -

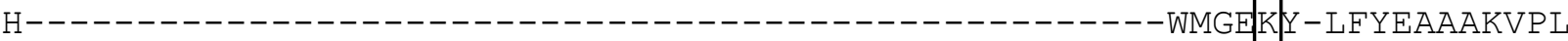
H-- - - - - - - - - - - - - - - - - - N---

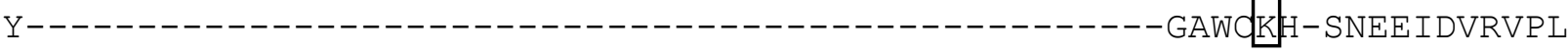

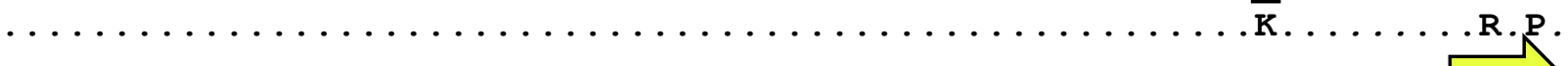

\section{$\beta 6$}

LAFW-PGH----IAPGVTH-ELASS-LDLLPTLAALAGAP--LPN---------VTLDGFDLSPLL-LGT LVRY-PRL---SRQGAISH-AFATV-MDVTPTLLDLAGVR--HP-GKRWRGREIAEPRGRSWLGWL-SGE IIKY-GKH---LPQGMVSD-TPVYG-LDWMPTLAKMMNFK--LPTD-------RTFDGESLVPVL-EQK LFHW-PKH---VPAGKRFS-HPVSA-LDLYPTFAALAGAK--VEEN--------QHLDGTNMWPAF-IKN IVRW-PGV---VKPDTKCD-KYLII-EDFYPS ILEMAQVK--HYKT-------VQPIDGISFIPLL-KQT MIAG-PGI---APG--LHL-TPTSN-LDVTPTLADLAGISLEEVR---------PWTDGVSLVPMV-NGV IFAG-PGI---KQQKKPVDHLLTQPTLDLLPTLCDLAGIA--VPA---------EKAGISLAPTLRGEK IIRY-PKA---IKAGSTSS-AISMN-VDFAPTFLDFAGVE--VP---------SDIQGASLKPVL-ENE IVKY-PGH---VAPGSRCD-VPVTT-PDFYPTFLELAGVP--OKSG-------IPIDGRS IVPLL-AGN IVFD-PRV---NKHQDISE--MVLN-IDVTQTIADLAGVK--AP---------ESWQGKSLLPLV-KQE IIYD-PRPEADATRGSVCD-ALVES-IDLAPTFVEAAGGK--PAM--------HILEGESLIPIL-HGA IIYD-PSDKADATRGTVSD-ALVEM-IDLAPTFVDYAGGV--PPM--------HILEGKSLLPLL-HDD ILSW-PDQ---IKPRKSDP-LMIAF-ADLYPTLLSMMGFSKEIP---------ETVQTFDLSNEV-LTG MFRW-PGK---LTP-RQDD-LLFSA-PDIYPTLFGLMGLEELIP---------DTVEGTNFAKTV-SGI IVSRETSYK-GRVAGKTSD-ALVEN-VDIFPTLVELCGLE--GP----------KTDGKS I LQVI-DRP

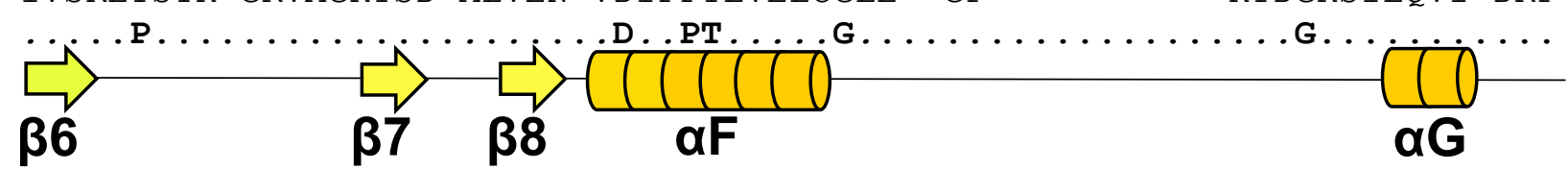


lauk 348 GKS---PRQSLFFYPSYPDE-----------VRGVFAVRTGKYKAHFFTQGSAHSDTTADPACHASSSLT

1hdh 446 TEA---AHDENTVTGWE--------------LFGMRAIRQGDWKAVYLPA------------------PV

3ed4 371 ALK---REKPLIFGIDMPFQDD---------PTDEWAIRDGDWKMIIDRNN------------------

6bia 348 ENP--HKDEPIYALRHRKG-------------YSDAAIRMNQWKALKVNQ-------------------

6USS 415 GDP---SKGRSLYWNFPNHWGNDG-----PGIGPTCTVRKGDWKLIYYYEN-------------------

4ug4 377 ER-----TEPVLMEYAAEAS-----------YAPLVAIREGKWKYVYCAL--------------------

5 gt 354 QKK---SHPYVVSEWHSEYEYV---------TTPGRMVRGPRYKYTHYLEG------------------- 


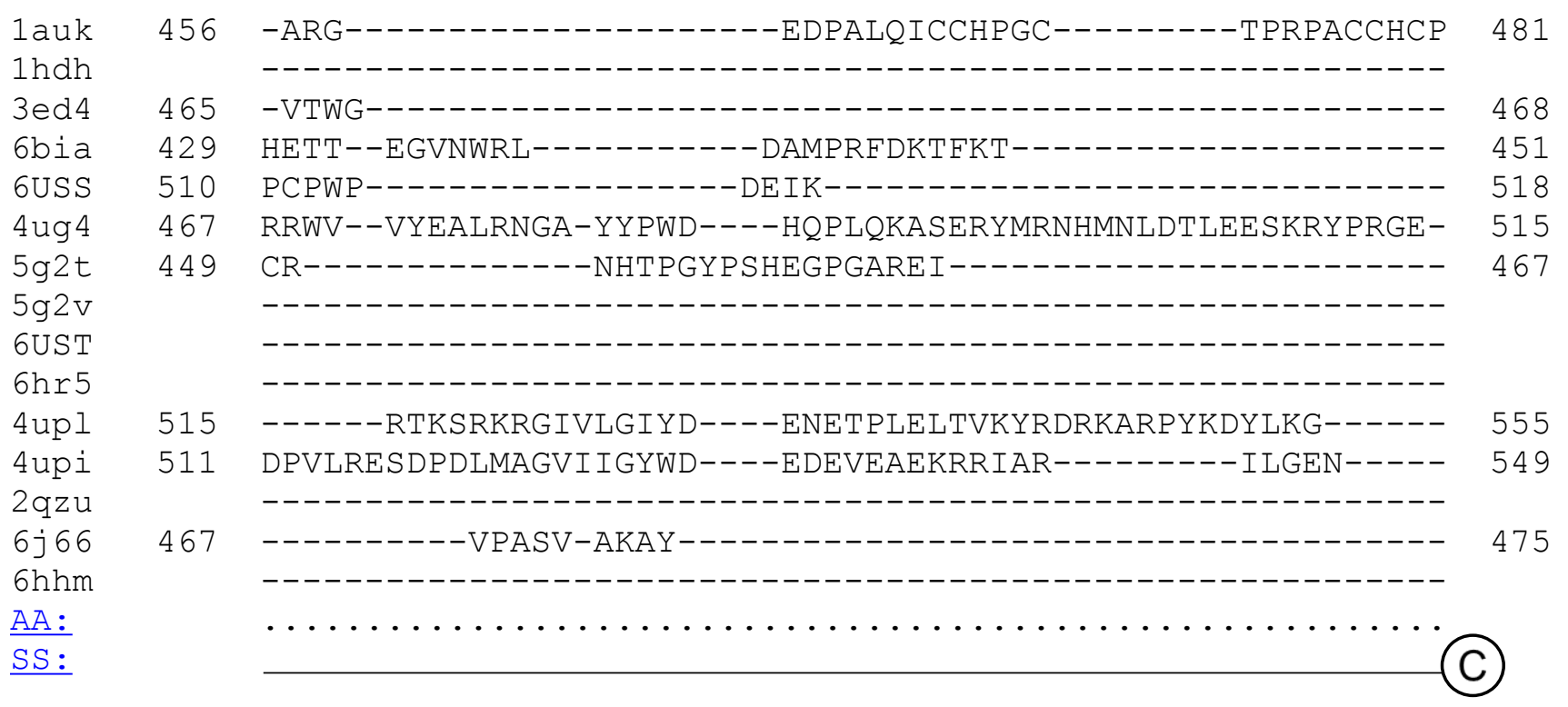

Figure S4. Multiple Sequence Alignment and Secondary Structural Features of Extant Microbial Sulfatases. Loops 2 and 3 are annotated in red and blue, respectively. Residues used to generate rubric are in bolded purple. Conserved active site residues are boxed. Secondary structural elements conserved between the enzymes are indicated (SS), as well as completely conserved residues (AA). See also Figure 5G. 
A

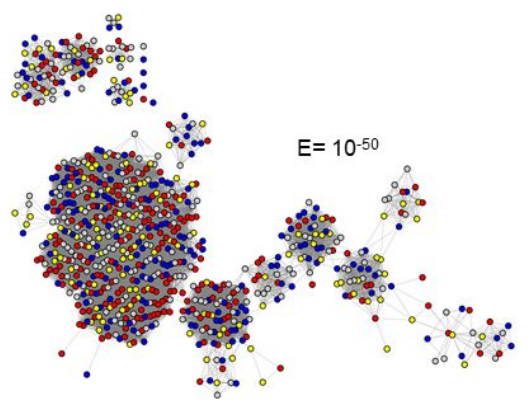

C

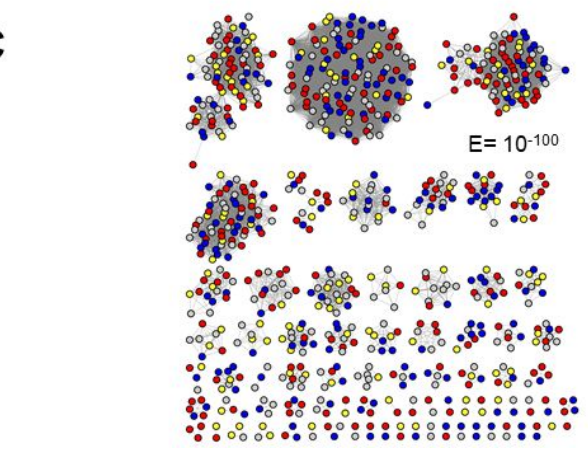

B

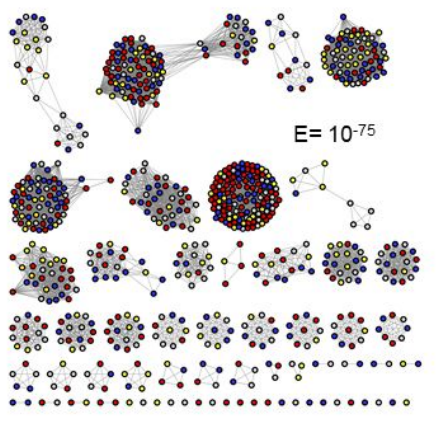

Loop 1

Loop 2

Loop 1,2

No Loop

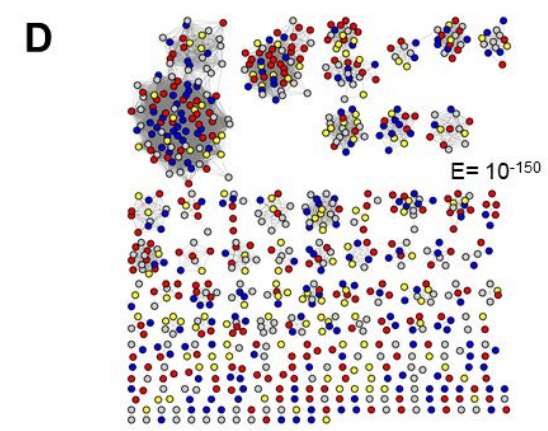

Figure S5. Sequence Similarity Networks of HMP Sulfatase Enzymes. Enzymes are clustered using varying alignment scores and colored by presence of Loop 2 (red), Loop 3 (blue), both Loop 2 and Loop 3 (yellow) or No Loop (grey). 
A

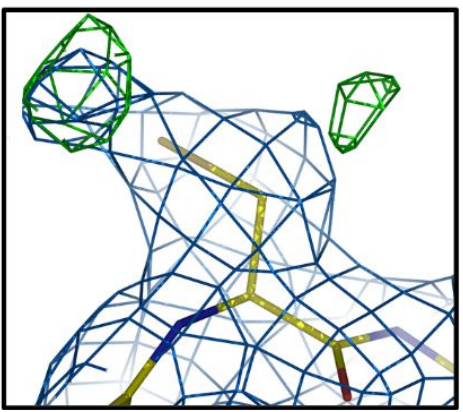

B

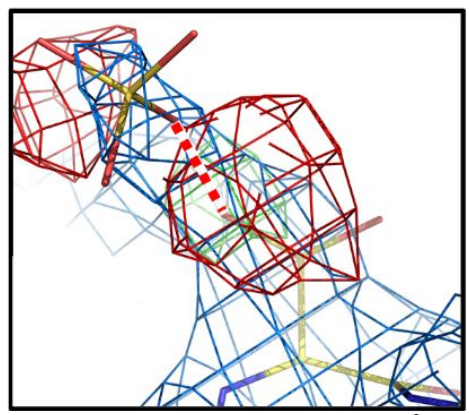

C

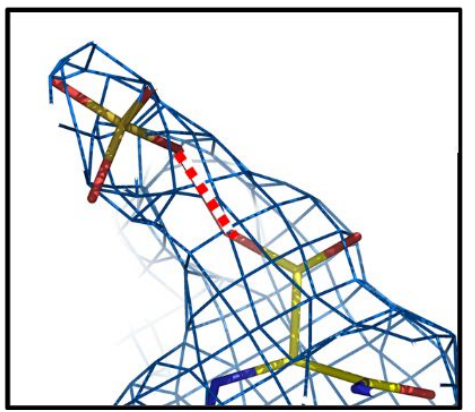

Figure S6. Electron Density at the Active Site of the $2.5 \AA$ Resolution Structure of BfSulf. $2 \mathrm{~F}_{\mathrm{o}}-\mathrm{F}_{\mathrm{c}}$ density in blue, positive $\mathrm{F}_{\mathrm{o}}-\mathrm{F}_{\mathrm{c}}$ density in green, and negative $\mathrm{F}_{\mathrm{o}}-\mathrm{F}_{\mathrm{c}}$ density in red. Dashed red line indicates covalent bond. Densities contoured at 1.5 o. A. With Cys-88 at 100\% occupancy, positive difference density is evident. B. With an fGly-hydrate/gem-diol covalently linked to a sulfate ion at 100\% occupancy, negative density is evident. C. Electron density is satisfied by an fGly-hydrate/gem-diol covalently linked to a sulfate ion at $20 \%$ occupancy. 


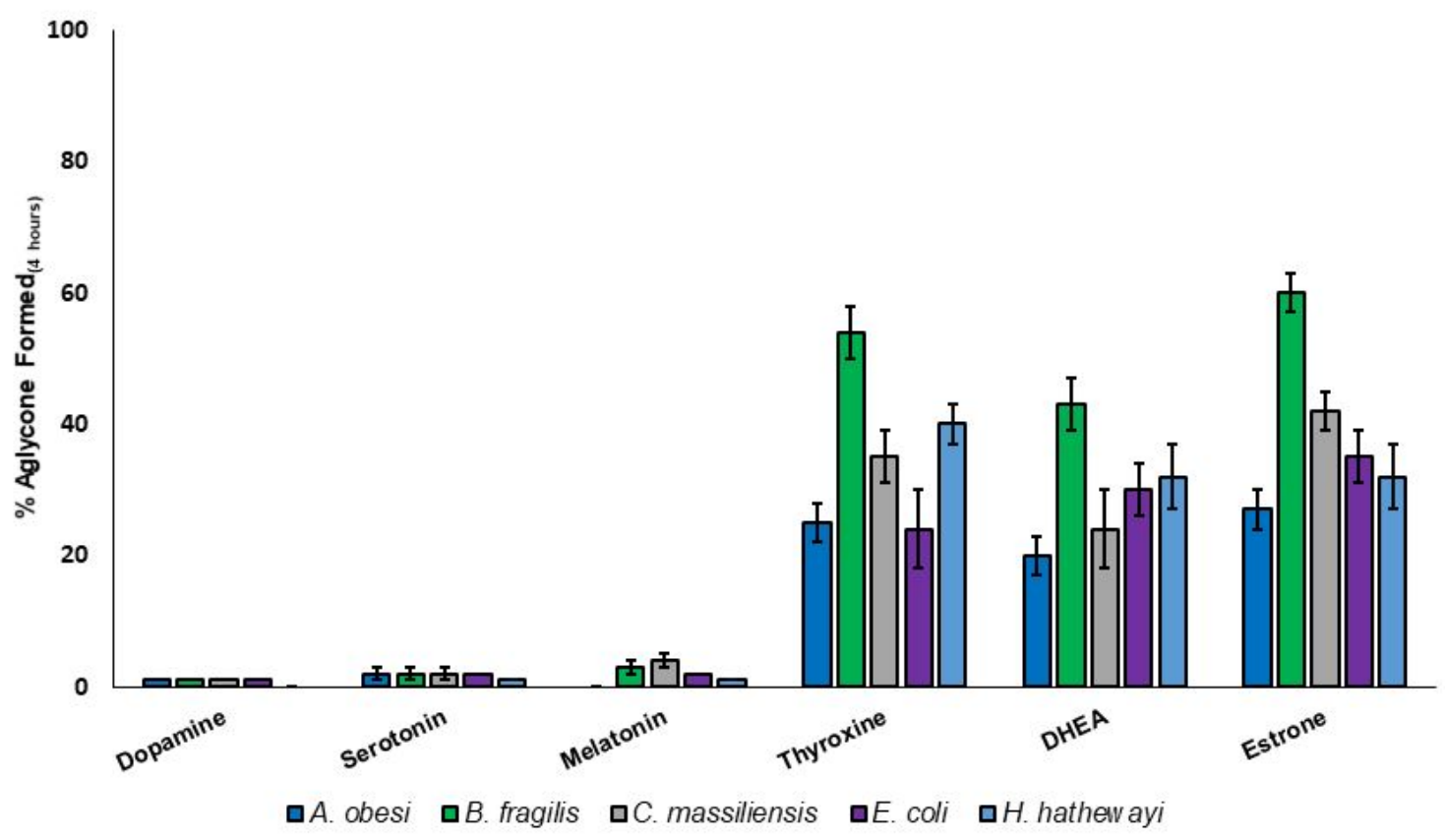

Figure S7. Percent Aglycone formed after 4-hour Sulfatase Incubation. 


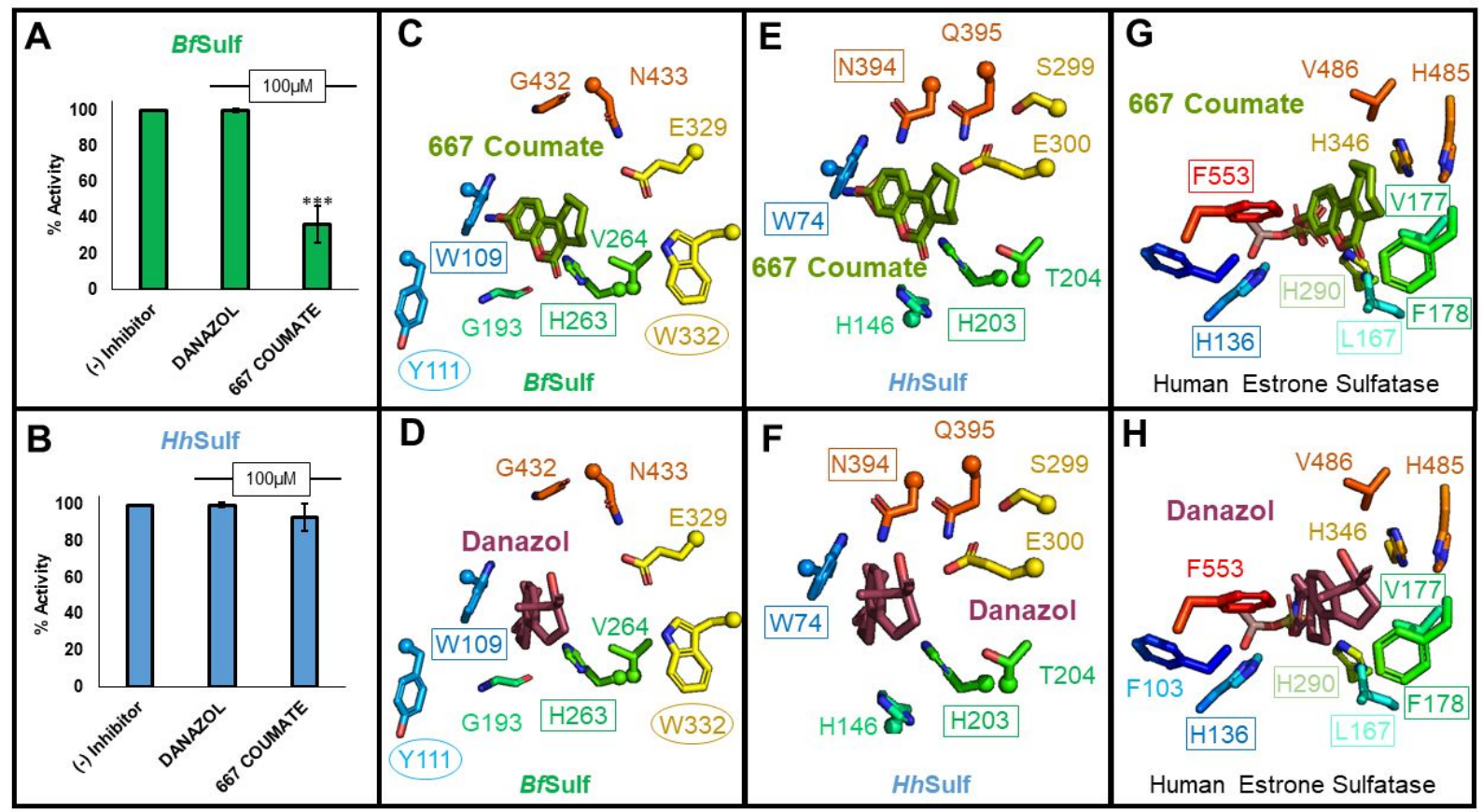

Figure S8. Inhibition of Gut Microbial Sulfatases. A. Percent activity of BfSulf after addition of $100 \mu \mathrm{M}$ inhibitor.; *** $=\mathrm{P}<0.001$. B. Percent activity of $H$ hSulf after addition of $100 \mu \mathrm{M}$ inhibitor. C. 667 Coumate docked in BfSulf active site; residues contacting the ligand are boxed, with additional aromatic residues in oval. D. Danazol docked in BfSulf active site; residues contacting the ligand are boxed, with additional aromatic residues in oval. E. 667 Coumate docked in $H h$ Sulf active site; residues contacting the ligand are boxed. F. Danazol docked in $H h$ Sulf active site; residues contacting the ligand are boxed. G. 667 Coumate docked in Human Estrone Sulfatase (1P49) active site; residues contacting the ligand are boxed. H. Danazol docked in Human Estrone Sulfatase (1P49) active site; residues contacting the ligand are boxed. Schrodinger Glide Dock scores are listed in Table S11. 

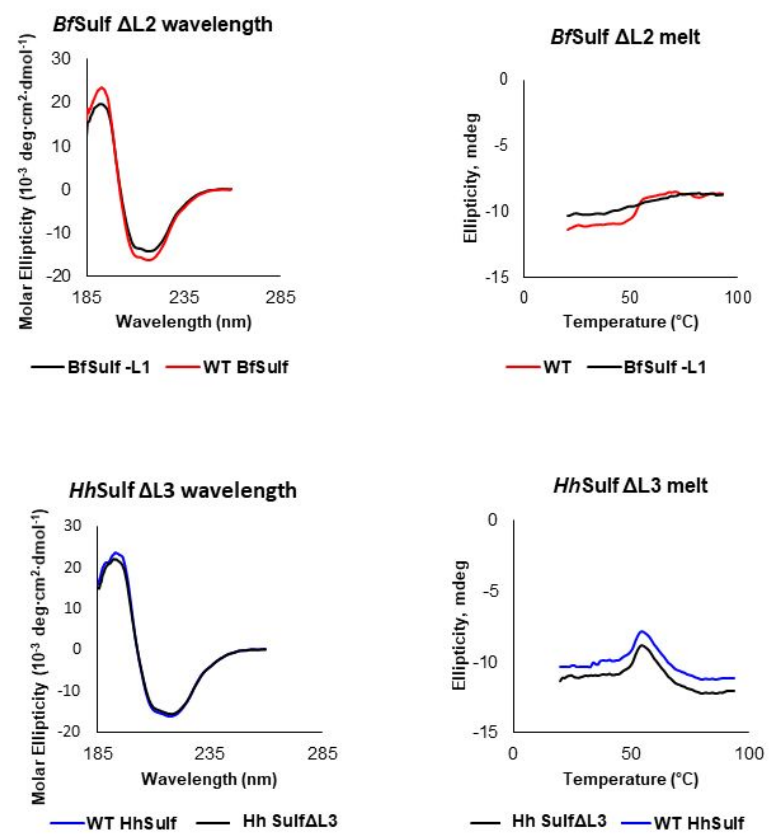

Figure S9. CD Scans. Top: BfSulf $\Delta \mathrm{L} 2$ wavelength and melt scans. Bottom: $H h$ Sulf $\Delta \mathrm{L} 3$ wavelength and melt scans. 